\title{
Factor XIII Val34Leu polymorphism in patients with cardiac syndrome $\mathrm{X}$
}

\author{
Gamze Babur Güler ${ }^{1}$, Ulaankhuu Batgerel², Ekrem Güler ${ }^{3}$, Oğuz Karaca ${ }^{3}$, \\ Çetin Geçmen², Hacı Murat Güneş ${ }^{2}$, Hicaz Zencirkıran Aguş², \\ Ali Metin Esen ${ }^{2}$, Mehmet Muhsin Türkmen ${ }^{3}$ \\ ${ }^{1}$ Istinye State Hospital, Istanbul, Turkey \\ ${ }^{2}$ Kartal Kosuyolu Training and Research Hospital, Cardiology Clinic, Istanbul, Turkey \\ ${ }^{3}$ Medipol University, Faculty of Medicine, Istanbul, Turkey
}

\begin{abstract}
Background: The aim of the study was to examine the frequency of factor XIII polymorphism among patients with cardiac syndrome $X(C S X)$.

Methods: This study was designed as a cross-sectional and observational study. Forty-eight female patients with CSX and 36 controls matched by age, gender, diabetes, and hypertension were studied. CSX was defined as typical chest pain during rest or effort, abnormal test result for exercise ECG, and presence of angiographically normal epicardial coronary arteries after ruling out inducible spasm. Factor XIII gene polymorphism was investigated by using CVD Strip Assay (ViennaLab Diagnostic GmbH) commercial kit.

Results: The frequency of factor XIII (Val/Leu + Leu/Leu) mutation was significantly higher in patients with CSX (43\%) than in controls (19\%) $(p=0.02)$. Frequency of the Leu allele was significantly higher in the patient group (23.5\% vs. $11.1 \%, p=0.04)$. Factor XIII (Val/ $/$ Leu + Leu/Leu) mutation $(p=0.01, O R=3.42 ; 95 \%$ CI 1.22-9.58) and smoking $(p=0.04$, $O R=3.33,95 \%$ CI 1.05-10.58) were identified as independent predictors of the disease in multivariate regression analysis.

Conclusions: This study indicates that there is an evidence for association between factor XIII Val34Leu polymorphism and CSX. (Cardiol J 2014; 21, 1: 6-10)
\end{abstract}

Key words: cardiac syndrome $\mathrm{X}$, factor XIII polymorphism

\section{Introduction}

Cardiac syndrome $\mathrm{X}(\mathrm{CSX})$ is a clinical entity of angina-like chest pain and positive response to cardiac stress test without coronary artery disease (CAD). Studies showed that the prognosis of CSX, which is considered in the class of stable angina pectoris, was generally better than the prognosis of the patients with acute coronary syndrome and patients detected to have a disorder in coronary angiography [1, 2]. Myocardial ischemia due to coronary microvascular dysfunction and increased sensitivity to pain are the mechanisms predominantly considered to be responsible for the pathophysiological process, while reasons such as insulin resistance, estrogen deficiency, and autonomous dysfunction are also being discussed for etiology [3].

Factor XIII (FXIII) as a plasma transglutaminase, plays a role in the stabilization of fibrin which

Address for correspondence: Gamze Babur Güler, MD, Istinye State Hospital, Istanbul, Turkey, e-mail: gamzebabur@hotmail.com

Received: 17.02.2013 Accepted: 19.04.2013 
is an important step of the coagulation cascade. FXIII Val34Leu polymorphism occurs as a result of the substitution of amino acid leucine (Leu) for valine (Val) at position 34 of the FXIII gene [4]. Several studies have demonstrated that this polymorphism was protective against $\mathrm{CAD}$, as well as myocardial infarction $[5,6]$. However, its relation to CSX was not investigated. In this study, our aim is to examine the frequency of FXIII polymorphism among patients with CSX who are known to have an overall good prognosis.

\section{Methods}

This study was designed as a cross-sectional and observational study. Forty-eight female patients diagnosed with CSX who were admitted to Kartal Koşuyolu Advanced Training and Research Hospital and a control group of 36 women were recruited into the study between March 2008 and May 2009, after the approval of local ethics committee.

CSX was defined as typical chest pain during rest or effort, abnormal test result for exercise ECG, and presence of angiographically normal epicardial coronary arteries. Control group was selected from volunteers presented to our hospital with the complaint of atypical angina who had normal result from exercise ECG and had similar risk profile with the patients (diabetes, hypertension, age, gender).

Patients who had abnormal echocardiography findings (dilated cardiomyopathy, hypertrophic cardiomyopathy, valvular cardiac disease, congenital heart disease, left ventricular hypertrophy, right ventricular hypertrophy) or history of peripheral vascular disease, chronic renal failure, hepatic failure, and thyroid dysfunction were excluded from the study. All exclusion criteria were applied also to the control group. Both patient and control groups were clinically examined and evaluated with rest ECG.

Both patient and control groups had 2-dimensional and Doppler echocardiography according to the recommendations of American Society of Echocardiography [7], patient exclusion criteria were evaluated.

Whole blood samples were obtained in EDTA tubes to study FXIII gene polymorphism. Total genomic DNA from peripheral whole blood samples of the patients were isolated with the GenXtract solution supplied with the kit. After that, target DNA regions were amplified with multiplex polymerase chain reaction by using biotin-labeled primers. Amplified products were then checked in agarose gel $3 \%$. Following the detection of the amplicons of the related genes, $\mathrm{PCR}$ products were hybridized to test strip containing nucleotide probes specific to the immobilized allele on the nitrocellulose membrane by using CVD Strip Assay (ViennaLab Diagnostic $\mathrm{GmbH}$ ) commercial kit. Hybridization process was performed in Profiblot T48 (Tecan) hybridization device. Sequences labeled with hybridized biotin were detected by using streptavidin-alkaline phosphatase and color substrates.

\section{Statistical analysis}

Mean \pm standard deviation was used for continuous variants, while percentages were used for categorical variants. Unpaired t test was used to test the difference between the continuous variants which showed normal distribution between patient and control groups.

Normal distribution was tested with one sample Kolmogorov-Smirnov test. Pearson $\chi^{2}$, Fisher's exact test and Continuity Correction (Yate's Correction) test were used to test the categorical variants. The variants for which evident significance $(p<0.1)$ were found in the univariate analysis were included in multivariate logistic regression analysis to determine the independent components of CSX. $\mathrm{P}$-value of $<0.05$ was considered significant for all tests. Statistical Package for the Social Sciences (SPSS version 11.0, SPSS Inc., Chicago, IL, USA) was used.

\section{Results}

The characteristics of the patients participating in the study are shown in Table 1 . Accordingly, there was no difference between patient and control groups except for the history of smoking $(\mathrm{p}=0.04)$.

\section{Genotype and allele frequency of FXIII Val34Leu polymorphism among healthy control group}

$\mathrm{Val} / \mathrm{Val}, \mathrm{Val} / \mathrm{Leu}$ and Leu/Leu genotypes were detected in $29(80 \%), 6(16 \%)$ and $1(2 \%)$ of the patients, respectively. Allele frequencies were $88.9 \%$ and $11.1 \%$ for Val and Leu, respectively (Table 2).

\section{Genotype and allele frequency of FXIII Val34Leu polymorphism among patients with CSX}

$\mathrm{Val} / \mathrm{Val}, \mathrm{Val} / \mathrm{Leu}$ and Leu/Leu genotypes were shown in 27 (56\%), 19 (39\%) and 2 (4\%) of the patients with CSX, respectively. Allele frequencies were $76.5 \%$ and $23.5 \%$ for Val and Leu, respectively (Table 2). 
Table 1. General characteristics of patients and controls.

\begin{tabular}{lccc}
\hline & Patients $(\mathbf{n}=\mathbf{4 8})$ & Controls $(\mathbf{n}=\mathbf{3 6})$ & P \\
\hline Age [year] & $53 \pm 6$ & $54 \pm 8$ & $0.57^{*}$ \\
Body mass index $\left[\mathrm{kg} / \mathrm{m}^{2}\right]$ & $29.83 \pm 3.90$ & $31.19 \pm 4.11$ & $0.13^{*}$ \\
Hypertension [\%] & 41 & 44 & $0.97^{* *}$ \\
Diabetes [\%] & 20 & 22 & $1^{* *}$ \\
Smoking [\%] & 33 & 13 & $0.04^{* *}$ \\
Hyperlipidemia [\%] & 38 & 35 & $0.92^{* *}$ \\
\hline
\end{tabular}

*Unpaired student $t$ test was used; ${ }^{* *}$ Fisher's Exact test was used; ${ }^{* *}$ Continuity Correction test was used

Table 2. Frequency of Val-Leu allele and genetic variations.

\begin{tabular}{lccc} 
& Patients (n) & Controls (n) & P \\
\hline Val/Val & $56 \%(27)$ & $80 \%(29)$ & $0.06^{*}$ \\
Val/Leu & $39 \%(19)$ & $16 \%(6)$ & \\
Leu/Leu & $4 \%(2)$ & $2 \%(1)$ & $0.02^{* *}$ \\
Val/Leu + Leu/Leu & $43 \%(21)$ & $19 \%(7)$ & Odds ratio $=3.22,95 \% \mathrm{Cl} \mathrm{1.18-8.78}$ \\
Val & $76.5 \%$ & $88.9 \%$ & $0.04^{* *}$ \\
Leu & $23.5 \%$ & $11.1 \%$ & Odds ratio $=2.453,95 \% \mathrm{Cl} 1.02-5.86$ \\
\hline
\end{tabular}

*Pearson $\chi^{2}$ test was used; **Fisher's Exact test was used; Val — valine; Leu — leucine

Table 3. Univariate analysis for major risk factor in cardiac syndrome $\mathrm{X}$.

\begin{tabular}{lcc}
\hline & $\begin{array}{c}\text { Odds ratio } \\
(95 \% \mathrm{CI})\end{array}$ & $\mathrm{P}^{*}$ \\
\hline Diabetes & $0.9(0.32-2.63)$ & 1 \\
Hypertension & $0.8(0.37-2.13)$ & 0.97 \\
Smoking & $3.1(1.01-9.49)$ & 0.04 \\
Factor XIII mutation & $3.2(1.18-8.78)$ & 0.02 \\
(Val/Leu + Leu/Leu) & & \\
Hyperlipidemia & $0.8(0.35-2.10)$ & 0.92 \\
\hline
\end{tabular}

${ }^{*} \chi{ }^{2}$ test; $\mathrm{Cl}$ - confidence interval; Val — valine; Leu — leucine

Frequency of Leu allele was significantly higher in patients with CSX compared to the control group ( $23.5 \%$ vs. $11.1 \%$; $\mathrm{p}=0.04, \mathrm{OR}=2.453$, 95\% CI 1.02-5.86).

Multivariate analysis model, which was generated according to the results of the univariate analysis in the aim to determine the predictors of CSX disease, included smoking and FXIII polymorphism Table 3 . As a result, both FXIII polymorphism $(\mathrm{Val} / \mathrm{Leu}+\mathrm{Leu} / \mathrm{Leu})(\mathrm{p}=0.01, \mathrm{OR}=3.42,95 \%$ CI 1.22-9.58) and smoking ( $\mathrm{p}=0.04$, OR $=3.33$, $9.5 \%$ CI 1.05-10.58) were found predictors of the
Table 4. Multivariate analysis for independent predictors.

\begin{tabular}{|c|c|c|c|}
\hline & Beta & $\mathbf{P}$ & $\begin{array}{c}\text { Odds ratio } \\
\text { (95\% CI) }\end{array}$ \\
\hline $\begin{array}{l}\text { Factor XIII mutation } \\
\text { (Val/Leu + Leu/Leu) }\end{array}$ & 1.23 & 0.01 & $\begin{array}{c}3.42 \\
(1.22-9.58)\end{array}$ \\
\hline Smoking & 1.20 & 0.04 & $\begin{array}{c}3.33 \\
(1.05-10.58)\end{array}$ \\
\hline
\end{tabular}

Hosmer and Lemeshow Test: $p=0.525 ;$ Nagelkerke $R^{2}$ : 0.155; $\mathrm{Cl}$ - confidence interval; Val - valine; Leu — leucine

disease, while FXIII polymorphism was a stronger predictor (Table 4).

\section{Discussion}

In our study, Leu allele frequency was identified significantly higher in patients with CSX than the control group, and FXIII Val34Leu polymorphism was found to be the most important factor as an independent predictor of the disease.

Lack of a standard diagnostic criteria and consensus on pathophysiology along with inefficient treatment options make CSX a despairing disease for both patients and physicians. In order to explain 
the pathophysiological abnormality, various theories such as abnormal coronary flow reserve [8], insulin resistance [9], enhanced red cell sodium-hydrogen exchange [10], and abnormal cardiac sensitivity [11] were suggested. The most proven and accepted ideas are endothelial dysfunction and inflammation, and increased sensitivity to pain $[3,12]$. Endothelial dysfunction reduces the release of anti-inflammatory and anti-thrombogenic factors and reduces the availability of nitric oxide by disrupting the vasoconstriction/vasodilatation balance [13]. Endothelial dysfunction has also a prognostic value since patients with chest pain having normal or almost normal coronary arteries showed increased frequency of cardiovascular events during follow-ups [14]. Long-term follow-up results of the patients with CSX were reported as highly good due to the lack of major acute coronary events (as cardiac death and acute myocardial infarction) [1], however the persistence of the symptoms, reduced quality of life and frequent hospital admissions due to inefficient treatments led to discussions on good prognosis.

In fact, the risk profile of this patient group is similar to the patients with occlusive CAD (conventional cardiovascular risk factors, increased inflammation, endothelial dysfunction etc.). Investigations on mechanisms are ongoing to understand why these patients do not have critical atherosclerotic lesions in coronary arteries and do not develop acute coronary events despite having evidence on coronary microvascular dysfunction. Studies are focused on platelet reactivity to explain this good prognosis $[15,16]$. Platelet reactivity following exercise or mental stress was measured in normal subjects, patients with CAD and patients with CSX, and it was demonstrated that reactivity was increased in $\mathrm{CAD}$ patients, remained the same in normal subjects and decreased in patients with CSX [17]. These findings put vascular protection and potential protective factors against acute thrombotic events on the agenda.

FXIII, which is also called a fibrin stabilizing factor due to its essential role in maintaining homeostasis, is an enzyme effective in the last step of the coagulation cascade. FXIII is a zymogen with tetrameric (A2B2) structure which consists of 2 active A subunits and 2 inhibitory B subunits, and is found in plasma as a pro-enzyme [18]. FXIII forms cross-links between fibrin monomers by transglutaminase activity making the clot more resistant to fibrinolysis [19]. Congenital FXIII is a rare autosomal recessive disorder resulting in hemorrhagic diathesis. It is characterized by subcu- taneous and intramuscular hematomas, intracranial hematomas, and defective wound healing [20]. The most frequent mutation in the genes coding for the FXIII is G-T transition in the exon of the gene coding for FXIIIA [4]. This mutation causes valine to convert to leucine in A subunit (FXIII AL) [4]. This mutation is defined as FXIII Val34Leu polymorphism due to high prevalence among population [4]. As a result of this mutation, thrombin activation of FXIII-A subunit and stability of fibrin will be decreased, and alternatively its interaction with other coagulation factors will be affected [6].

The studies focused on the assumption that the presence of FXIII Leu34 allele might have protective effects against arterial and venous thrombosis [21-23]. In a study including 398 patients with acute coronary syndrome and 196 healthy control subjects, it was demonstrated that FXIII Leu incidence was significantly low in the patient group and suggested that this polymorphism was protective against acute coronary syndrome [6]. Kohler et al. [6] concluded that the mutation in FXIII, a fibrin stabilizer, would lead to ineffective cross-linking and formation of weaker fibrin structures. In our study, both homozygote and heterozygote polymorphism of Leu34 allele was identified frequently in the patient group compared to the control group. CSX occurrence increases in individuals with Leu allele, whereas the position of this mutation in the benign prognosis of the disease may be associated with the ineffective function in the coagulation cascade of FXIII.

Smoking is believed to contribute to etiology similarly to other risk factors of CAD due to possible endothelial dysfunction [24]. Therefore, smoking secession is recommended together with reducing the atherosclerotic risk factors at the beginning of the non-pharmacological treatment of the disease [25]. Our study also identified smoking as an independent predictor for CSX together with FXIII mutation, supporting the literature.

CSX is considered among subgroups of stable $\mathrm{CAD}$ and it is emphasized that it has a better prognosis than the patient group with proven occlusive coronary lesion. We think that the presence of this mutation in CSX may be associated with a pathophysiological process in which microvascular bed is affected, while macrovascular bed is preserved.

It is the first study on patients with CSX relating FXIII Val34Leu polymorphism. A limitation to our study is the small number of patients. Large studies conducted on great number of patients will clarify the effect of this polymorphism on the pathogenesis and prognosis of CSX. 


\section{Conclusions}

This study indicates that there is an evidence for association between factor XIII Val34Leu polymorphism and CSX.

\section{Conflict of interest: none declared}

\section{References}

1. Kaski JC, Rosano GM, Collins P et al. Cardiac syndrome X: Clinical characteristics and left ventricular function. Long-term follow-up study. J Am Coll Cardiol, 1995; 25: 807.

2. Kemp HG, Kronmal RA, Vlietstra RE, Frye RL. Seven year survival of patients with normal or near normal coronary arteriograms: A CASS registry study. J Am Coll Cardiol, 1986; 7: 479.

3. Singh M, Singh S, Arora R, Khosla S. Cardiac syndrome X: Current concepts. Int J Cardiol, 2010; 142: 113-119.

4. Anwar R, Gallivan L, Edmonds SD, Markham AF. Genotype/ /phenotype correlations for coagulation Factor XIII: Specific normal polymorphisms are associated with high or low Factor XIII specific activity. Blood, 1999; 93: 897-905.

5. Wartiovaara U, Perola M, Mikkola H et al. Association of FXIII Val34Leu with decreased risk of myocardial infarction in Finnish males. Atherosclerosis, 1999; 142: 295-300.

6. Kohler HP, Stickland MH, Ossei-Gerning N, Carter A, Mikkola H, Grant PJ. Association of a common polymorphism in the factor XIII gene with myocardial infarction. Thromb Haemost, 1998; 79: 8-13.

7. Gottdiener JS, Bednarz J, Devereux R et al. American Society of Echocardiography recommendations for use of echocardiography in clinical trials. J Am Soc Echocardioraphy, 2004; 17: 1086-1119.

8. Vesely MR, Dilsizian V. Microvascular angina: assessment of coronary blood flow, flow reserve, and metabolism. Curr Cardiol Rep, 2011; 13: 151-158.

9. Jadhav S, Ferrell W, Greer IA, Petrie JR, Cobbe SM, Sattar N. Effects of metformin on microvascular function and exercise tolerance in women with angina and normal coronary arteries: A randomized, double-blind, placebo-controlled study. J Am Coll Cardiol, 2006; 48: 956-963.

10. Koren W, Koldanov R, Peleg E, Rabinowitz B, Rosenthal T. Enhanced red cell sodium-hydrogen exchange in microvascular angina. Eur Heart J, 1997; 18: 1296-1299.
11. Pasceri V, Lanza GA, Buffon A, Montenero AS, Crea F, Maseri A. Role of abnormal pain sensitivity and behavioral factors in determining chest pain in syndrome X. J Am Coll Cardiol, 1998; 31: 62-66.

12. Hurst T, Olson TH, Olson LE, Appleton CP. Cardiac syndrome $\mathrm{X}$ and endothelial dysfunction: New concepts in prognosis and treatment. Am J Med, 2006; 119: 560-566.

13. Davignon J, Ganz P. Role of endothelial dysfunction in atherosclerosis. Circulation, 2004; 109 (23 suppl. 1): III27-III32.

14. Bugiardini R, Manfrini O, Pizzi C, Fontana F, Morgagni G. Endothelial function predicts future development of coronary artery disease: A study of women with chest pain and normal coronary angiograms. Circulation, 2004;109: 2518-2523.

15. Lanza GA, Sestito A, Iacovella $S$ et al. Relation between platelet response to exercise and coronary angiographic findings in patients with effort angina Circulation, 2003; 107: 1378-1382.

16. Lanza GA, Andreotti F, Sestito A, Sciahbasi A, Crea F, Maseri A. Platelet aggregability in cardiac syndrome X. Eur Heart J, 2001; 22: 1924-1930.

17. Sestito A, Maccallini A, Sgueglia GA et al. Platelet reactivity in response to mental stress in syndrome $\mathrm{X}$ and in stable or unstable coronary artery disease. Thromb Res, 2005; 116: 25-31.

18. Siebenlist KR, Meh DA, Mosesson MW. Plasma factor XIII binds specifically to fibrinogen molecules containing chains. Biochemistry, 1996; 35: 10448-10453.

19. Bereczky Z, Katona E, Muszbek L. Fibrin stabilization (factor XIII), fibrin structure and thrombosis. Pathophysiol Haemost Thromb, 2004; 33: 430-437.

20. Egbring R, Kröniger A, Seitz R. Factor XIII deficiency: Pathogenic mechanisms and clinical significance. Semin Thromb Hemost, 1996; 22: 419-425.

21. Franco RF, Pazin-Filho A, Tavella MH, Simoes MV, Marin-Neto JA, Zago MA. Factor XIII Val34Leu and the risk of myocardial infarction. Haematologica, 2000; 85: 67-71.

22. Gemmati D, Serino ML, Ongaro A et al. A Common mutation in the gene for coagulation factor XIII-A (Val34Leu): A risk factor for primary intracerebral hemorrhage is protective against atherothrombotic diseases. Am J Hematol, 2001; 67: 183-188.

23. Catto AJ, Kohler HP, Coore J, Mansfield MW, Stickland MH, Grant PJ. Association of a common polymorphism in the factor XIII gene with venous thrombosis. Blood, 1999; 93: 906-908.

24. Virdis A, Giannarelli C, Neves MF, Taddei S, Ghiadoni L. Cigarette smoking and hypertension. Curr Pharm Des, 2010; 16: 2518-2525.

25. Banks K, Lo M, Khera A. Angina in women without obstructive coronary artery disease. Curr Cardiol Rev, 2010; 6: 71-81. 